\title{
BACKGROUND AND INITIAL EXPERIMENTS WITH THE DYNAMIC ERGODIC DIVERTOR ON TEXTOR
}

\author{
K. H. FINKEN, $*^{*}$ S. S. ABDULLAEV, $\dagger$ M. F. M. DE BOCK, $\ddagger$ B. GIESEN, $\dagger$ \\ M. VON HELLERMANN, $\ddagger$ G. M. D. HOGEWEIJ,$\$$ M. JAKUBOWSKI, $\dagger$ R. JASPERS, \\ M. KOBAYASHI,$\dagger$ H. R. KOSLOWSKI,$\dagger$ M. LEHNEN,$\dagger$ G. MATSUNAGA, $\dagger$ O. NEUBAUER,$\dagger$ \\ A. POSPIESZCZYK, $\dagger$ U. SAMM,$\dagger$ B. SCHWEER, $\dagger$ and R. WOLF $\dagger$ \\ $\dagger$ Institut für Plasmaphysik, Forschungszentrum Jülich GmbH, EURATOM Association \\ Trilateral Euregio Cluster, D-52425 Jülich, Germany \\ $¥$ FOM-Institute for Plasma Physics Rijnhuizen, Association EURATOM-FOM, Trilateral Euregio Cluster \\ P.O. Box 1207, NL-3430 BE Nieuwegein, The Netherlands
}

Received April 29, 2004

Accepted for Publication August 8, 2004

Recently, the Dynamic Ergodic Divertor (DED) with 18 helically wound coils at the high field side has been installed on TEXTOR. The DED allows static and dynamic operation up to $10 \mathrm{kHz}$. The specific features of ergodization and the open laminar zone are discussed. The dynamic feature leads to induced electrical currents and to a force transfer from the external coils to the plasma. The structures due to the DED near field are described, which result in a stripelike pattern seen both in the light of recycling particles $\left(H_{\alpha}\right.$, impurities $)$ and in the heat deposition pattern. The ergodization leads either to an enhanced plasma rotation-probably due to edge electric fields-or to a reduction of the central rotation if a tearing mode is excited; the result depends on the sense of DED rotation.

KEYWORDS: ergodic structures, power distribution, induced tearing modes

\section{INTRODUCTION}

The formation of an ergodic layer can be an interesting means of destroying the good confinement properties of closed flux surfaces at special locations. Such a location of interest is the boundary of the plasma: The power-with the exception of the radiated fractionleaves the plasma conductively or convectively by passing through a relatively thin boundary layer. In order to spread the heat to a larger area, the Dynamic Ergodic Divertor $^{1-3}$ (DED) has been developed. The DED cre-

\footnotetext{
*E-mail: k.h.finken@fz-juelich.de
}

ates an ergodic boundary layer of the plasma and thus potentially allows for a wider power deposition pattern, an improved screening of impurities, and an enhanced particle removal by the pump limiter advanced limiter test-II (ALT-II).

The expression "dynamic" refers to a rotating perturbation magnetic field imposed by the DED coils. For the rotation, different frequencies are foreseen: At a few hertz, the heating pattern of the divertor strike zone is smeared out over the large area of the divertor target plate; at frequencies up to $10 \mathrm{kHz}$, locked modes can be unlocked, or a differential rotation in the plasma edge can be imposed with hopefully favorable effects on confinement. It is estimated that the rotating DED current generates a torque at the plasma edge.

\section{DED PROGRAM OBJECTIVES}

The main objectives of the DED program concern the following:

1. reduction of heat load by distributing the heat over large areas by multiple and rotating strike points

2. influencing of plasma transport, in particular impurities, by ergodization of the edge magnetic field and diverting the plasma flow to the target plates in the near field with the objective of impurity screening and optimized radiation cooling

3. active influence of plasma transport by externally induced rotational shear with the possibility of triggering transport barriers. This will be complemented by heating and current drive experiments, changing heating composition, momentum transfer, and current profile. 
4. exploration of the applicability of external rotating magnetic fields for the suppression of magnetohydrodynamic (MHD) instabilities. This includes stabilization of neoclassical tearing modes, error field amplification and rotation damping, and investigation of resistive wall modes. The aim is to extend the operational limits, also in conjunction with local heating and current drive [e.g., electron cyclotron resonance heating (ECRH)].

5. validation of three-dimensional transport models for the description of complex edge plasmas with ergodic zones and island structures, also forming an interdisciplinary link to stellarator physics

6. The fundamental properties of stochastic plasmas might also help to understand transient phenomena, such as edge-localized modes ${ }^{4}$ (ELMs) and disruptions.

A major international community has been formed that is interested in one or several of these aspects and which has indicated its interest of participation. For the exchange of knowledge in the field, a new workshop (SEP Workshop: Stochasticity in Fusion Edge Plasmas) has been installed and will be held biannually.

\section{THE EXPERIMENTAL SETUP}

The DED, as shown in Fig. 1, is a new conceptual approach that recently has been installed in TEXTOR (Refs. 5 through 10); however, it is based on previous theories and experiments on ergodization. ${ }^{11-17}$ The main component of the DED is a set of magnetic perturbation coils whose purpose is to ergodize the magnetic field structure in the plasma edge region; these coils are located inside the vacuum vessel at the high field side (HFS) of the torus. The set consists of 16 individual coils (4 quadruples) plus 2 compensation coils. The individual perturbation coils follow the direction of the equilibrium magnetic field of the plasma edge helically once around the torus. The maximum currents amount to $15 \mathrm{kA} /$ coil.

The main perturbation modes $(m=10 \ldots 14, n=4)$ are centered at $m / n=12 / 4$; this mode structure has been selected because it creates only small local perturbations (magnetic islands) and avoids undesired disturbances in the plasma core. The DED has the unique feature that the perturbation field is not static as in most other devices but that it has the option of rotation. This is achieved by supplying the DED coils with a four-phase alternatingcurrent similar to what is done for an induction motor. To our knowledge only the tokamaks CSTN and HYPTOC (Refs. 18 through 21) at Nagoya University have similar features and-at low perturbation current levels-also the TEXT (Ref. 22) tokamak. The DED can be operated direct-current around $50 \mathrm{~Hz}$ or at 7 frequencies in the band from 1 to $10 \mathrm{kHz}$ (Refs. 23 through 30).

\section{THEORETICAL BACKGROUND}

\section{IV.A. Magnetic Perturbations and Formation of Ergodic Layer}

The perturbation coils create nonaxisymmetric magnetic field perturbations at the plasma boundary. These magnetic perturbations are resonant to the several rational magnetic flux surfaces $\psi_{m n}$ determined by $q\left(\psi_{m n}\right)=$ $m / n$, where $q(\psi)$ is the safety factor, $\psi$ is a toroidal flux labeling magnetic surface, and $m$ and $n$ are poloidal and toroidal mode numbers, respectively. The behavior of magnetic surfaces under resonant magnetic perturbations can be conveniently described by field line equations in the Hamiltonian form:

$$
d \psi / d \varphi=\partial \psi_{p} / \partial \vartheta, \quad \partial \vartheta / d \varphi=-\partial \psi_{p} / \partial \psi,
$$

where $\psi_{p}=\psi_{p}(\psi, \vartheta, \varphi)$ is the poloidal flux:

$$
\begin{aligned}
\psi_{p}(\psi, \vartheta, \varphi)= & \int \frac{d \psi}{q(\psi)}+\varepsilon \sum_{m} h_{m}(\psi) \\
& \times \cos (m \vartheta-n \varphi+\Omega t) .
\end{aligned}
$$

The first term on the right side of Eq. (2) describes unperturbed field lines $\vartheta=\varphi / q(\psi)$ lying on magnetic surfaces $\psi=$ const. The second term describes the resonant magnetic perturbations with the Fourier coefficients $h_{m}(\psi)$ corresponding to the $(m, n)$-resonant modes, the parameter $\varepsilon \approx \delta B_{r} / B_{t}$ stands for the dimensionless magnitude of perturbation, and $\Omega$ is the frequency of the rotating magnetic field. The magnetic perturbation is localized on the HFS $(\vartheta=\pi)$ of torus with a poloidal extension of about $\Delta \theta \approx 70 \mathrm{deg}$ ( $80 \mathrm{deg}$ with compensation coils). In the standard operational regime the toroidal mode number $n=4$.

The determination of Fourier harmonics $h_{m}(\psi)$ via magnetic field perturbations and its properties are investigated in Ref. 31. The typical spectrum of $h_{m}(\psi)$ is shown in Fig. 2. It is localized near the central mode $m_{c}=m_{0} \xi$ with the width $\Delta m \approx \pi \xi / \Delta \theta$, where $m_{0} \approx 20$ and $\xi(<1)$ is the tangent of field lines on the HFS. The perturbation harmonics $h_{m}(\psi)$ strongly decay inward along the radial coordinate $r \sim \psi^{1 / 2}, h_{m}(\psi) \sim r^{m / \xi}$.

The formation of the ergodic zone of field lines in the presence of magnetic perturbation is a typical example of chaotic motion in Hamiltonian systems. ${ }^{32-36}$ Each resonant magnetic perturbation, $h_{m} \cos (m \vartheta-n \varphi+\Omega t)$, breaks the rational magnetic surface $\psi_{m n}, q\left(\psi_{m n}\right)=m / n$ forming a chain of magnetic islands with the widths $\Delta \psi_{m n} \sim \varepsilon^{1 / 2}$. At a certain level of magnetic perturbation $\varepsilon$ the magnetic islands start to overlap and create a zone of chaotic field lines. Typically the ergodic zone is formed by overlapping several magnetic islands $m: n$ with poloidal modes $m=10, \ldots, 14$. The contribution of modes $m<9$ at the corresponding interior resonant surfaces is small because of the strong radial decay of magnetic perturbations $h_{m}(\psi)$. Qualitatively the degree of ergodization is 


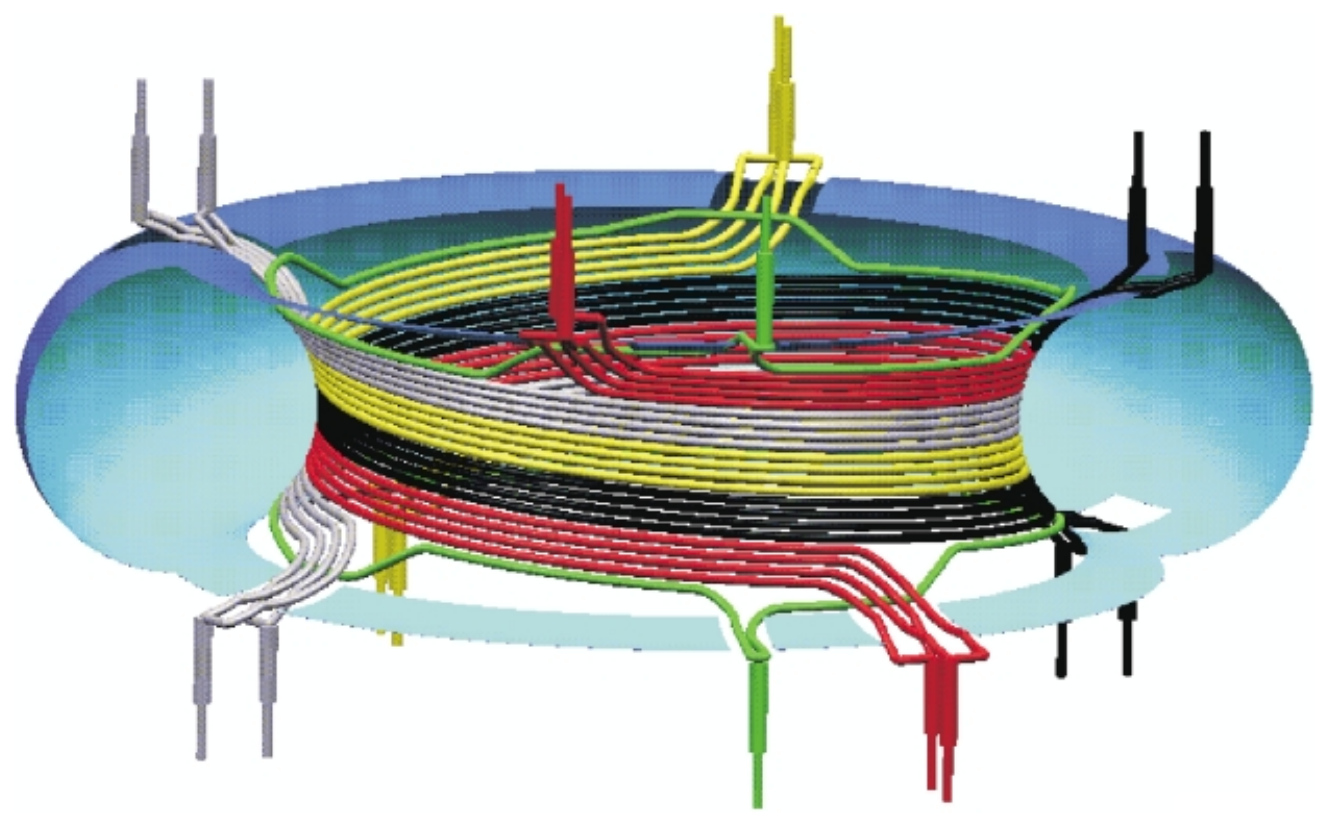

Fig. 1. Schematic drawing of the DED.

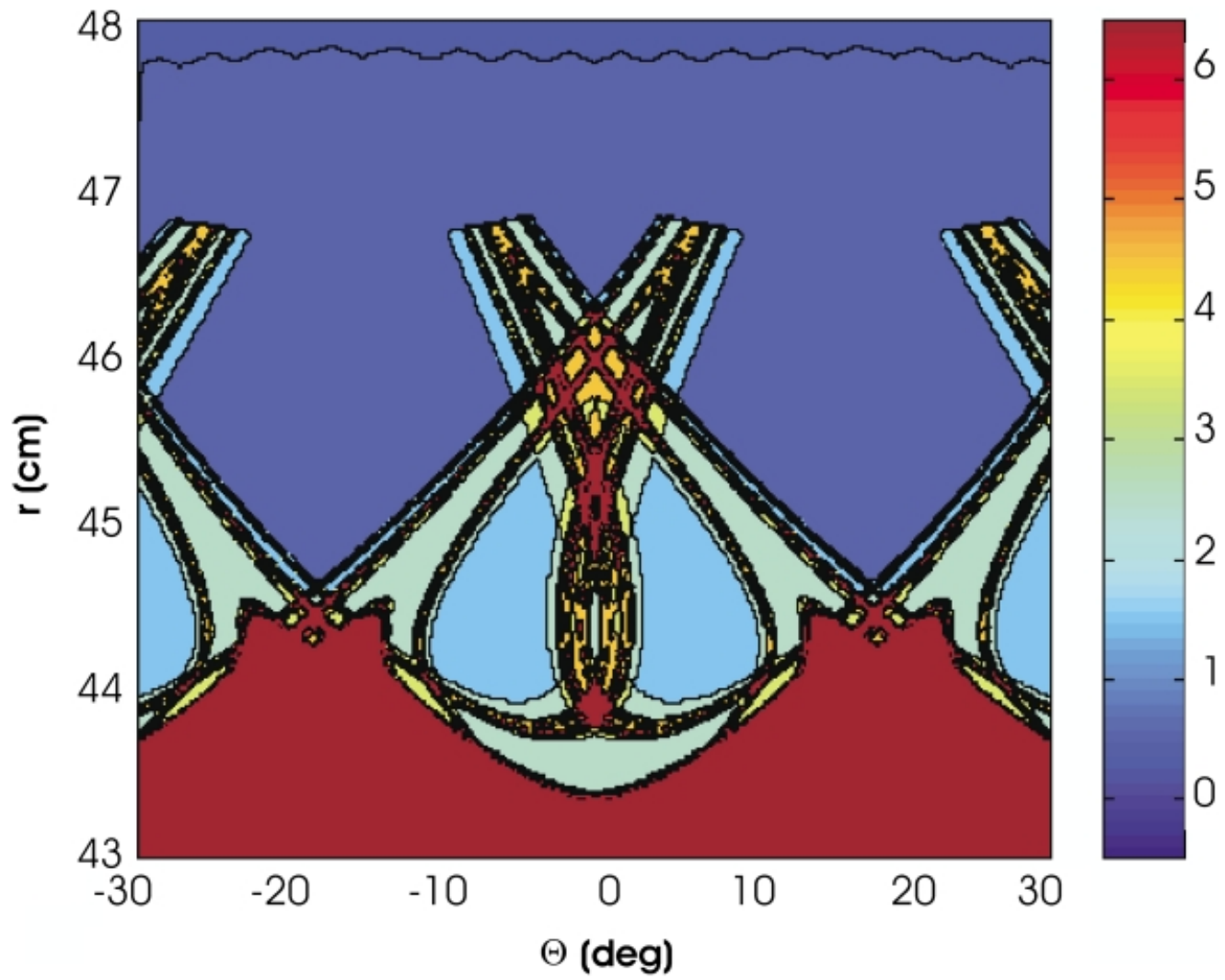

Fig. 3. Example of a laminar plot. The horizontal axis represents the poloidal angle while the vertical one shows the radius. The magnetic field lines of different connection lengths are plotted in different colors. Dark blue is the private flux zone, light blue and light green correspond to one and two poloidal turns, respectively, and red is the ergodic zone. 


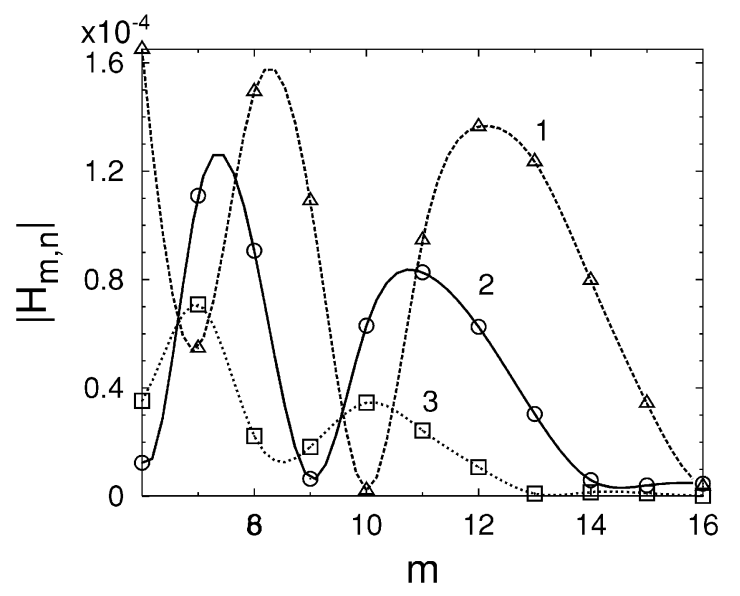

Fig. 2. Hamiltonian perturbation spectrum $\left|h_{m n}\right|(n=4)$ for different values of $\beta_{p o l}$ at the resonant magnetic surface $\psi_{m n}(m: n=12: 4)$ : Curve 1 corresponds to $\beta_{p o l}=$ 0.2 , curve 2 to $\beta_{p o l}=1$, and Curve 3 to $\beta_{p o l}=1.8$. The value of $h_{m n}$ is normalized to $B_{t}=2.25 \mathrm{~T}$ and refers to a DED current of $15 \mathrm{kA} /$ coil.

characterized by the Chirikov parameter, $\sigma=\left(\Delta \psi_{m+1, n}+\right.$ $\left.\Delta \psi_{m n}\right) / 2\left(\psi_{m+1, n}-\psi_{m n}\right)$, i.e., by the degree of overlapping neighboring magnetic islands. In the ergodic zone the value of $\sigma$ is $\sigma>1$.

The quantitative study of the ergodic zone of field lines is based on the field line tracing. We have developed an efficient mapping method to integrate Hamiltonian Eqs. (1) (Refs. 37 and 38) and applied it to study the chaotic magnetic field lines at the plasma edge in the presence of DED perturbations ${ }^{39-46}$ (see also a review ${ }^{47}$ as well as a mapping method for particles ${ }^{48}$ ). In Sec. IV.B we describe the main features of the structure of the ergodic zone obtained in these works.

\section{IV.B. The Structure of Ergodic and Laminar Zones}

The stochastization of field lines creates the region of open field lines at the plasma edge connecting to wall to wall. The region may be roughly divided into two zones: the ergodic and the laminar zones. The zone of field lines with large connection lengths (roughly more than six poloidal turns) can be defined as an "ergodic" zone, while the zone of field lines with a few poloidal turns defines the "laminar" zone. In addition, in particular for weakly ergodized systems, one finds magnetic islands with "intact" flux surfaces. The variation of these zones by changing the plasma parameters, for example, the plasma current or the toroidal magnetic field, allows one to study the different regimes of the plasma edge extended from the ergodic-dominated edge to those similar to normal divertor structures. There are relations to the island divertors of large helical devices such as W7-X or LHD, which in some operating regimes show weak ergodic zones as well.
The structure of the ergodic zone depends on the equilibrium plasma parameters $\beta_{p o l}$, the plasma current $I_{p}$, and toroidal magnetic field $B_{t}$. The level of ergodization can be controlled by varying these parameters. Particularly, the rational magnetic surfaces $\psi_{m n}$ can be shifted radially in the edge region by varying the plasma current or the toroidal magnetic field.

With increasing plasma current the resonant surfaces $\psi_{m n}(m=10, \ldots, 14 ; n=4)$ are moved toward the outside, closer to the DED coils. Highest ergodization levels with the Chirikov parameters $\sigma$ up to 6 are expected for the plasma currents around $600 \mathrm{kA}$. With increasing plasma current the fraction of field lines, which have smaller wallto-wall connection lengths, grows. This region is called a laminar zone. ${ }^{12,13,40,49-51}$ With the increase of plasma current the laminar zone grows while the ergodic zone becomes smaller. Because of its short connection lengths, the laminar zone is related to the scrape-off layer properties.

The properties of the laminar zone are best represented in a poloidal section at the plasma edge by contour plots of regions corresponding to different connection lengths of field lines. Such a contour plot, called a laminar plot, is shown in Fig. 3; the connection lengths of field lines are classified by the number of poloidal turns. The areas with a connection length of one poloidal turn are plotted in dark blue, that of two in light blue, and the areas with three or more poloidal turns successively in green, yellow, and red.

One of the important characteristics of open magnetic field lines is a pattern of field line striking points with divertor plates. This pattern, called magnetic footprints, mainly determines a heat and particle deposition to the wall. It consists of four pairs of helical stripes covering a finite poloidal extension on the HFS and continuously winding along the toroidal direction. The distance between the stripes in each pair grows with increasing plasma current. The study of a structure of each stripe similar to the laminar plot shows that it mostly consists of an area of field lines with one poloidal turn. Areas corresponding to field lines with two or more poloidal turns are successively smaller (see Refs. 28 and 40 for details). For this reason the areas with short connection lengths are most responsible for the final step of the transport of energy and particles to the wall. In this sense the laminar zone is similar to the scrape-off layer of a divertor tokamak.

\section{IV.C. Application of the EMC3-EIRENE Modeling to the DED Configuration}

In order to obtain quantitative predictions of the plasma in the boundary zone, several modeling efforts ${ }^{52-56}$ have been started. In a three-dimensional modeling, the inner radial boundary is at the flux surface just inside the $m=11$ islands. There, the flux boundary condition was imposed while the outer radial boundary is a few centimeters outside the divertor edge. Here, the decay lengths are specified as the boundary conditions. At the divertor 
surface, the Bohm condition was imposed, accelerating the plasma up to the sound speed, and the recycling flux proportional to the particle deposition pattern was taken into account by the EIRENE neutral particle Monte Carlo code.

Figure 4 shows the resulting $T_{e}$ profiles. It is found that the pattern of the high density and $T_{e}$ clearly matches the long connection flux tubes region, indicating that the flux tubes provide the loss channel of the plasma. The Mach number profile, on the other hand, was found to take the maximal at the short connection length flux tubes, where the acceleration of the plasma toward the divertor becomes highest. A rather high density is found in front of the divertor because of the recycling, as well as the strong poloidal modulation for both profiles. The ionization profile was found to have a strong correlation with the particle deposition pattern, affecting the density profile.

The energy deposition pattern onto the divertor is shown in Fig. 5. We obtained almost the same pattern for the particle deposition. The energy is mostly deposited poloidally in between the DED coil structure onto the divertor target plates, where the radial component of the perturbation field becomes strong. The one-turn flux tubes bring $80 \%$ of the energy flux to the divertor, although the hot plasma comes along the long flux tubes $(>180 \mathrm{~m})$.

The roles of the flux tubes with the different connection lengths may change depending on the magnetic field structure. The main parameters to control the structure are $\beta_{p o l}$ and the perturbation current. The value of $\beta_{p o l}$ enters into the degree of ergodization because at higher pressure the differential Shafranov shift increases the separation of the flux surfaces at the HFS and tends to shift inner flux tubes away from the DED coils, thereby weakening the DED field (the radial decay length of the DED field amounts to a few centimeters only for the $m / n=$ $12 / 4$ base mode). In addition, $\beta_{\text {pol }}$ modifies the pitch of the magnetic field lines, which results in a modification of the spectrum, which is taken with respect to the field lines. The high $\beta_{p o l}$ widens the ergodic zone and leads more of the longer flux tubes to the divertor plate than the case with the low $\beta_{p o l}$. The higher perturbation current would have the same effect as the high poloidal beta. At the moment it is observed, that with the $\beta_{p o l}=1$, most of the energy and particle flux are given to the short connection length flux tubes just before the deposition onto the divertor, while with the $\beta_{p o l}=0$ long connection length flux tubes still contribute substantially to the deposition process. The resulting effects on the transport properties will be studied in detail later.

\section{IV.D. Dynamic Aspects}

The high-frequency aspect of the DED field has been analyzed in cylindrical approximation. ${ }^{57-63}$ It has been shown that the "low-frequency" (relative to $\Omega_{i}$ ) electromagnetic wave of the DED effectively propagates in the area between the coils and the resonance layer as the compressional Alfvén wave (fast wave). ${ }^{64}$ At the resonance layer of the plasma different approximations have been made ${ }^{45}$ : The interaction layer is described by an annulus of finite resistivity, which is a function of the local electron temperature (skin effect). A theoretical uncertainty is the question of the radial width of this layer $(2 w)$. One reasonable choice (a) is the width of the ergodic zone derived from the Poincaré plots, which gives a typical value of $2 w=2.5 \mathrm{~cm}$; under a second assumption (b) the characteristic shielding width has been taken as the width of the statically calculated islands undiminished by overlapping (typically $0.5 \mathrm{~cm}$ ). This width is compatible with experiments made on TEXT (Ref. 22) for a situation of not fully ergodized edge field. Under a third assumption (c) a linear MHD theory has been applied, which results in a shielding thickness of less than a millimeter.

It has also been shown that the interaction of the external rotating field with the current driven in the shielding layer results in the transfer of angular momentum between DED coil and plasma. The maximum poloidal torque applied to the plasma amounts to about $100 \mathrm{Nm}$; this maximum occurs at a frequency that seems to depend mainly on the width of the current layer. In detail it depends on the assumed plasma temperature, on the applied frequency, and on the assumed island or ergodization width. Examples (a), (b), and (c) of the torque calculated for different conditions of the shielding current width are shown in Fig. 6. Even if a poloidal rotation of the plasma would be limited by neoclassical effects, the toroidal projection of the applied force is still somewhat larger than that imposed by tangential neutral beam injection (NBI) in TEXTOR. This beam injection generates a toroidal rotation of more than $10^{7} \mathrm{~cm} / \mathrm{s}$. A combined operation of the high-frequency DED with NBI will allow imposing interesting differential rotations between the plasma edge (action of the DED) and the core (by NBI).

\section{FIRST MEASUREMENTS}

Previous calculations suggest that the strongest action of the DED can be expected for (a) maximum DED currents, (b) for a plasma shifted to the HFS, (c) for plasma with a low value of the safety factor $q(a)$, (d) for a low value of $B_{t}$, and (e) for plasma with low $\beta_{p o l}$. For machine safety reasons, the initial values of the DED current are limited to $50 \%$ of the nominal amplitude. In this series of discharges, the plasma was shifted by 3 to $4 \mathrm{~cm}$ toward the DED, i.e., toward the HFS (Ref. 65). Typical pictures of the particle $\left(\mathrm{H}_{\alpha}\right)$ and power deposition pattern with active DED are shown in Fig. 7. Figure $7 \mathrm{a}$ is a toroidal view with a charge-coupled device (CCD) camera in the visible spectral range. Figure $7 \mathrm{~b}$ is a view on the divertor target plates (again a CCD 


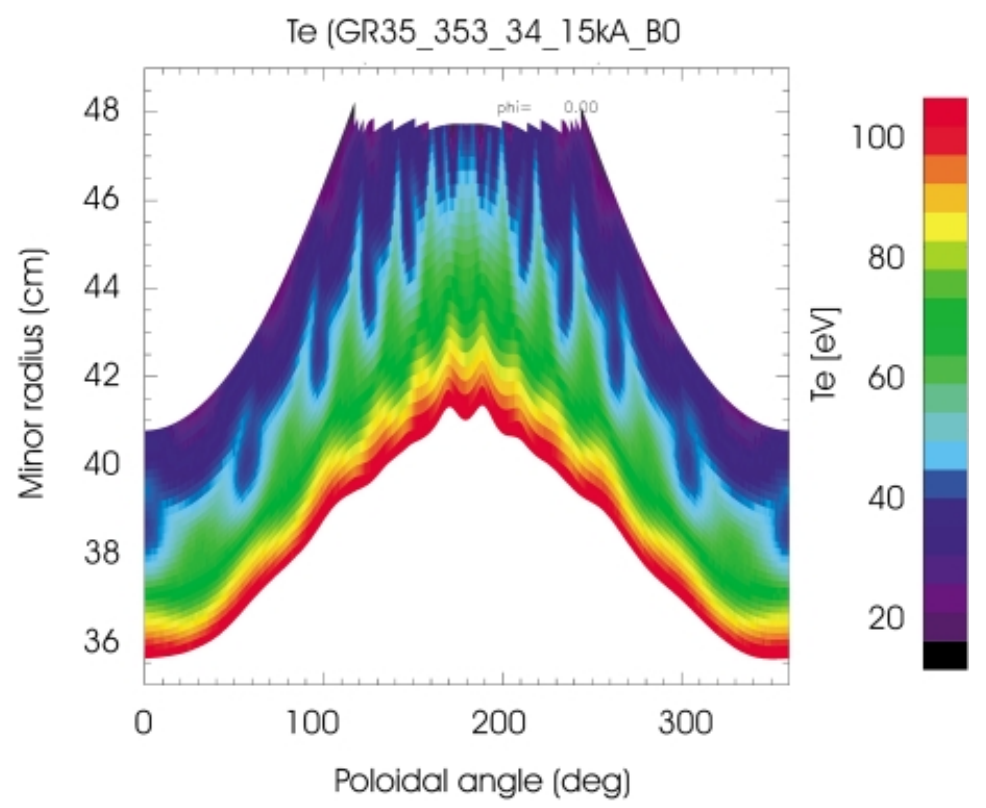

Fig. 4. $T_{e}$ profile at $\phi=0 \mathrm{deg}$ with the total recycling flux of $1.3 \times 10^{22}$ particles $/ \mathrm{s}(800 \mathrm{~A})$ and the input power of $1 \mathrm{MW}$ according to the three-dimensional transport modeling.

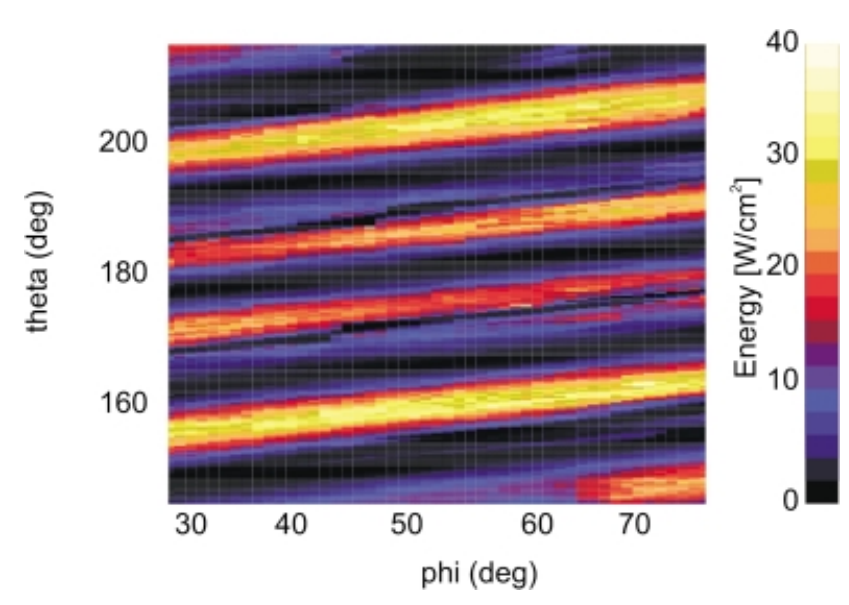

Fig. 5. Structure of the energy deposition pattern onto the divertor. The data result from the EMC3 modeling and correspond to the data of Fig. 4. camera), and Fig. 7c was taken by an infrared camera. The superposition of the equilibrium field of the plasma and the externally applied near field of the DED generates a characteristic stripe pattern in front of the DED coils. At a low ergodization level, one expects four rather broad stripes as shown in Fig. 7b, middle. These data are in agreement with the three-dimensional plasma transport modeling of the DED (Ref. 66). As one can easily see, the measured width of the stripes and their shape agree very well with the results from the modeling.

Local ion fluxes and electron temperatures are measured by a set of 18 Langmuir probes mounted in the tiles of the divertor target plate. For the measurement of these quantities, the DED pattern was slowly shifted; this was reached by programming the subsequent powering of the neighboring DED coils. This results in a rotation of the DED field of very low frequency (e.g., $2 \mathrm{~Hz}$ ) as it may be desired for a quasi-static analysis of localized data.

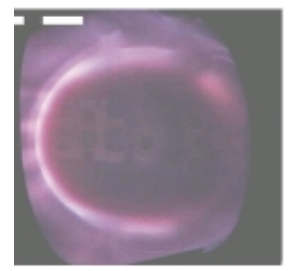

(a)

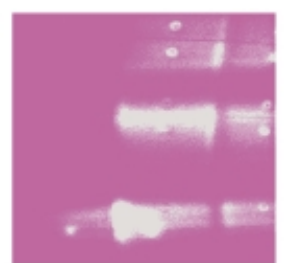

(b)

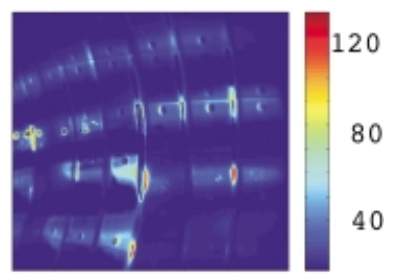

(c)

Fig. 7. Pattern induced by the action of the DED. (a) Toroidal view, (b) perpendicular view of a color CCD camera into TEXTOR, and (c) view by an infrared camera. The stripes in front of the DED coils are a prominent feature of the DED operation. The DED coils are "behind" the stripes, i.e., on (a) on the left side of the view and on the other figures running helically with the stripes. The near field of the DED coils imposes the stripe pattern. 


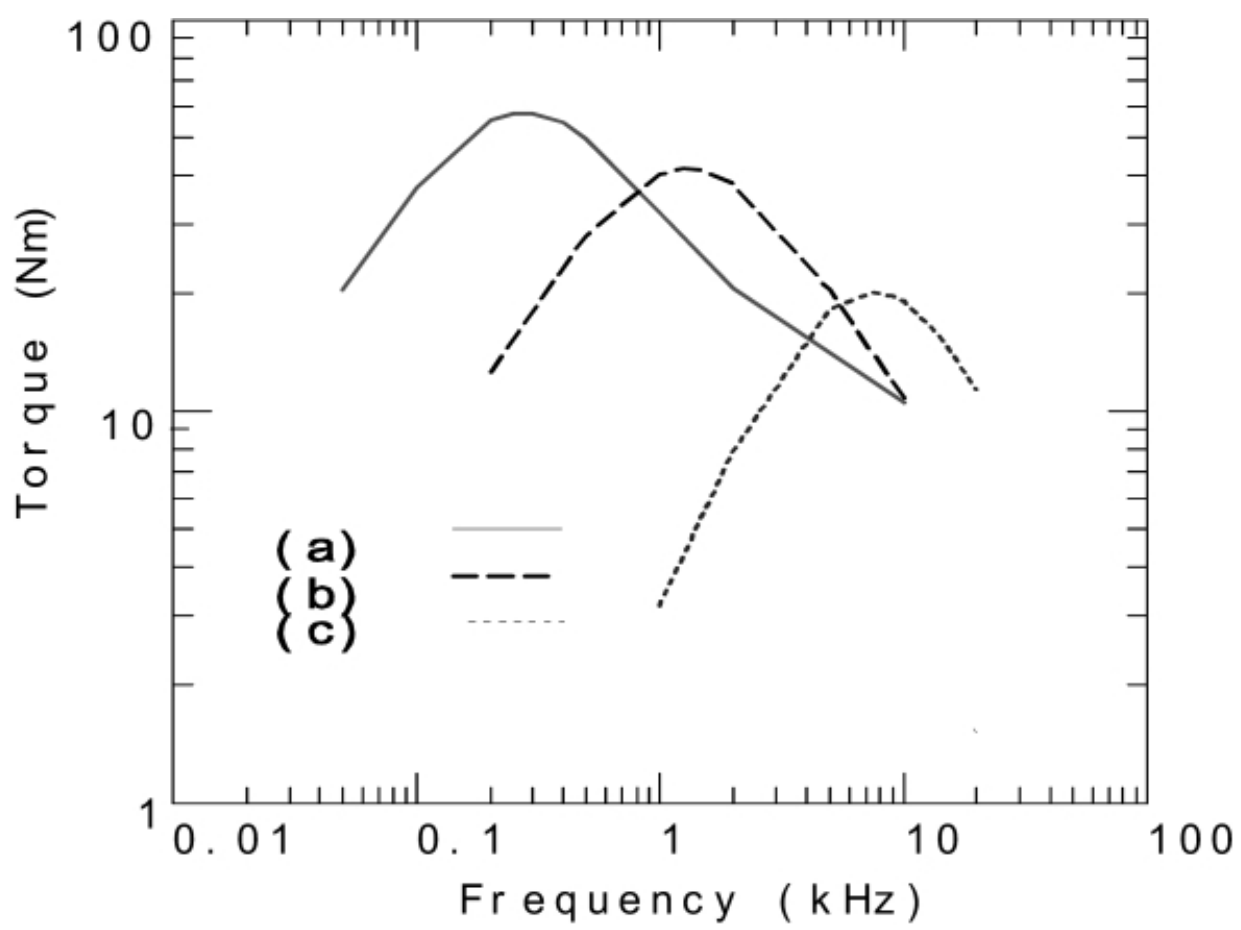

Fig. 6. Function of the transfer of torque from the external DED coils to the plasma at the resonant surface. Curves (a) and (b) represent the case of a skin-type shielding current with a width of the shielding layer of 2.5 and $0.5 \mathrm{~cm}$, respectively; for curve (c) an Alfvén resonance is assumed. The torque transfer for three assumptions of the shielding current thickness in cylindrical approximation ${ }^{57}$ is shown.

The static operation has shown that-as expectedthe heat flux is deposited along stripes in front of the DED coils. The dynamic operation of the DED has been foreseen to smear out the inhomogeneous heat flux to a large surface. A rough inspection by thermography confirms that this assumption is correct. The temperature development of a point of the divertor target surface has been recorded, and a Fourier analysis of this temperature yields the spectrum shown in Fig. 8. The curve represents the spectrum for the case of NBI and DED operated at about $2 \mathrm{kHz}$. As expected, it shows a pronounced peak at the DED frequency. Without dynamic DED operation, the curve is lower at frequencies below $1 \mathrm{kHz}$. This shows that - as expected - the heat smeared out by the rotating DED field.

After the first operation with the reduced current, each of the four neighboring DED coils was switched in parallel providing the $m / n=3 / 1$ scenario, which is characterized by the deep penetration of the perturbation field into the plasma. In this scenario the maximum current per coil is limited by the power supply to $3.75 \mathrm{kA}$ such that forces acting on the coils are no further limitation. Nevertheless, the total current per mode amounts to 30 $\mathrm{kA}$ because in the direct-current case eight neighboring coils carry the same current. This scenario provided many new features depending on the applied DED current and on the applied frequency. In particular, we distinguish two major cases ${ }^{67}$ : (a) the ergodization-dominated one and (b) the case with tearing mode excitation.

The ergodization-dominated scenario occurs at low DED current or for the corotating DED field up to the highest amplitudes. The $2 / 1$ tearing mode — and at higher

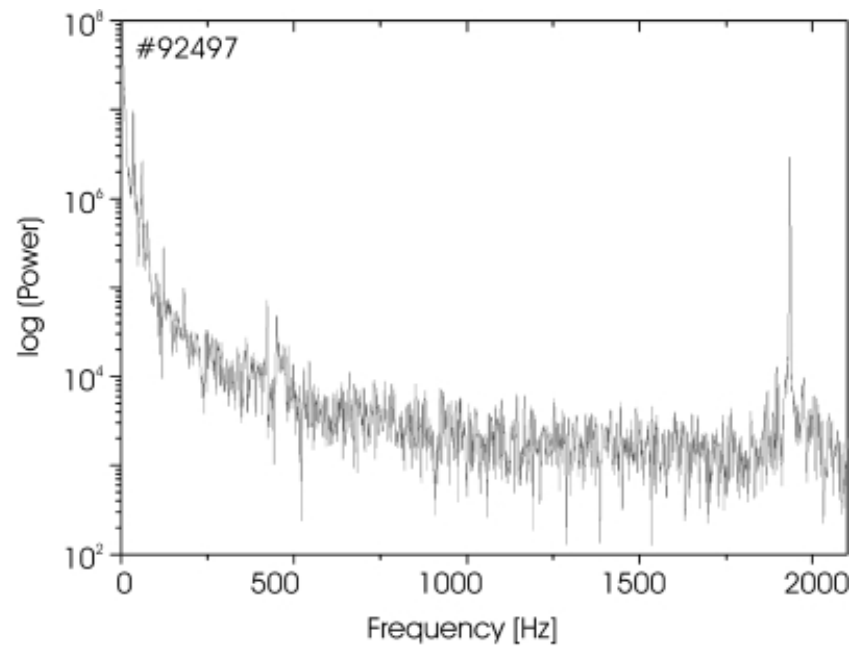

Fig. 8. Spectrum of the heat deposition for an NBI-heated plasma with additionally $2 \mathrm{kHz}$ DED rotation. 
DED current the $3 / 1$-is excited for the direct-current operation of the DED and for the counterrotating alternating-current case, provided that a rather reproducible current is exceeded. We define the corotating direction as the one in which the projection in the toroidal direction of the helical movement of the DED field is in the direction of the plasma current; the poloidal projection is in the ion diamagnetic drift direction. The counterrotation has just the opposite sense of rotation and opposite direction of the ponderomotive force, which we have treated above.

A remarkable result of the ergodization is the spinning up of the toroidal rotation, which is measured by charge-exchange recombination spectroscopy over twothirds of the minor radius, as one can see in Fig. 9. For the accessible conditions, the angular frequency of the plasma increases with about the third power of the applied DED current. The gain in rotation occurs over the whole measurable part of the plasma profile. Our present assumption is that the rotation results from a modification of the electric field at the plasma edge, which may be caused by the loss of electrons along the open magnetic field lines at the plasma edge in combination with the ambipolarity constraint. A modification of the electric potential by $200 \mathrm{~V}$ over a distance of $5 \mathrm{~cm}$ would explain both the direction of the induced rotation and its magnitude. It is interesting to note that the plasma acceleration is always in the same direction and independent of the DED rotation; actually, the rotation is in codirection even though at the interesting amplitudes the DED is counterrotating. Even though preliminary heat pulse propagation experiments indicate a reduction of the energy loss channel during the phase of high plasma rotation, an improvement of the energy confinement time has not yet been found.

The excitation of the $m / n=2 / 1$ tearing mode occurs rather reproducibly for a perturbation field amplitude of 5 to 10 gauss at the resonant surface, which corresponds to a modest DED current of 0.6 to $1.2 \mathrm{kA} /$ coil; in detail it depends on the heating scenario such as co- or counterNBI and ion cyclotron resonance heating. A preexisting tearing mode is locked at a much lower field. The tearing mode is locked with the DED field (i.e., static or rotating) and persists to the end of the discharge even if the DED is switched off.

The presence of the mode allows for a stable plasma operation only at values of $q(a)>4.5$, i.e., at a reduced plasma current. The presence of the mode has a strong influence on the plasma: It reduces-as expected-the

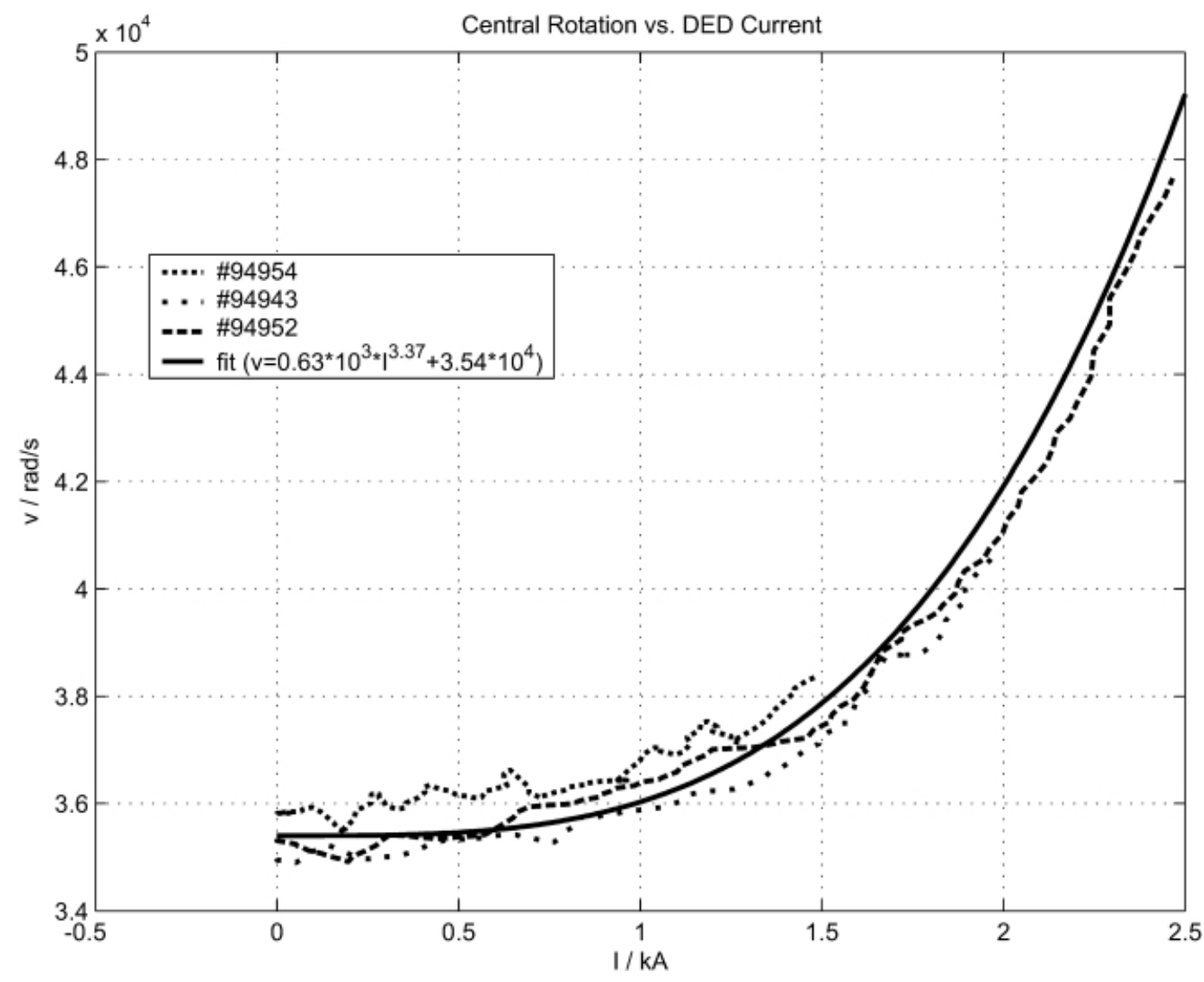

Fig. 9. Toroidal angular frequency as a function of the counterrotating DED current. 
particle and energy confinement by 10 to $20 \%$. The mode has a beneficial effect on the impurity purification of the plasma because the impurities are lost faster than the deuterium. The tearing mode also modifies strongly the toroidal plasma velocity, as one may see in Fig. 10. The dotted lines show the angular frequency profile before the onset of the mode and the full lines after the mode onset. One finds that at low DED current, i.e., before the onset of the mode, the plasma velocity spins up over the whole profile such as described as ergodization effect. When the mode sets in, the profile between the $q=1$ surface and the $q=2$ surface practically flattens. The stopping of the central rotation is so fast that it cannot be resolved.

\section{SUMMARY AND FUTURE DIRECTIONS}

With the DED, the TEXTOR group has installed a unique and very versatile experiment for influencing both the plasma edge and the core. For influencing the plasma edge only, it is foreseen to operate the DED predominantly in the fine $m / n=12 / 4$ mode while for a deeper penetration of the field a coarser mode is preferred, such as the $m / n=6 / 2$ or 3/1. Programmatic points of the fine mode are the understanding of edge ergodization and the investigation of a helical divertor. With the coarser mode, islands can be excited in a programmable way deep inside the plasma. These islands may act as seed islands and induce specific plasma reactions. A special point of interest is the interaction with these islands, e.g., by local heating with ECRH; they simulate the otherwise naturally excited tearing modes that limit the plasma performance. In case of a rotating DED field, the islands are-

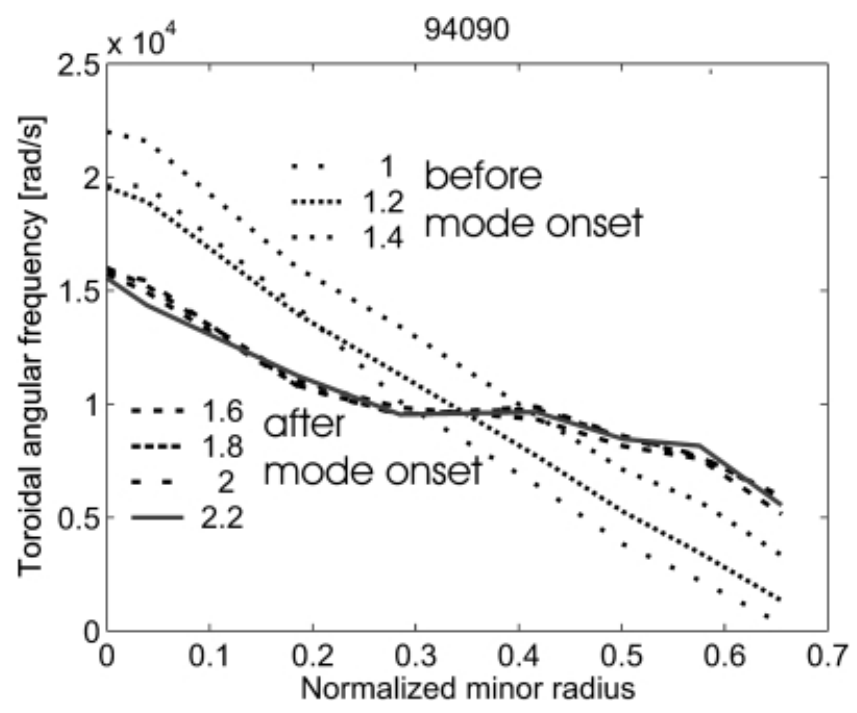

Fig. 10. Profile of the plasma rotation angular frequency before (dotted lines) and after (full lines) the onset of an $m / n=2 / 1$ tearing mode. according to theory- the key part where the force transfer between the external field and the plasma takes place. Therefore, they are of specific interest for questions of plasma acceleration or deceleration (locked modes or wall resistive modes), which play a key role for the plasma termination or the plasma confinement.

Beyond the closer topic, the work on the DED triggers the investigation of the more fundamental aspects of ergodization, which otherwise are not in the focus of interest of the fusion community. These basic aspects led to a collaboration with universities in the framework of a project in the "Sonderforschungsbereich 591." In addition, the considerations of ergodization led to new ideas of tackling disruptions and ELMs (Ref. 68); in particular the heat flux pattern during these events indicates the important role of ergodization for these events. ${ }^{69-71}$

A specific point of interest is the question of which role ergodization schemes can play in future devices. ${ }^{72}$ The focus here is on the mitigation of the effects of Type I ELMs, which are foreseen as the standard scenario for ITER. The first measurements stem from work at JFT-2M (Ref. 73) and were recently followed by work on DIII-D. On the Japanese device, the ELMs could be decreased partially on cost of the confinement quality while in DIII-D suppression of ELMs was observed without loss of confinement. ${ }^{74,75}$ These mitigation schemes require a static ergodization field only, which may still be implemented in ITER. If (slowly) rotating is tolerable, the ergodization can also be used to distribute the heat over an enhanced area. $^{76}$

\section{ACKNOWLEDGMENT}

This work was partially supported by the Sonderforschungsbereich 591.

\section{REFERENCES}

1. Special Issue: Dynamic Ergodic Divertor, Fusion Eng. Des., 37, 335 (1997).

2. K. H. FINKEN et al., J. Nucl. Mater., 220-222, 448 (1995).

3. K. H. FINKEN, Nucl. Fusion, 37, 583 (1997).

4. T. EICH et al., Phys. Rev. Lett., 91, 195003-1 (2003).

5. B. GIESEN et al., J. Nucl. Mater., 705 (1997).

6. O. NEUBAUER and U. BRAUNSBERGER, Proc. 17th IEEE/ NPSS Symp. Fusion Engineering, San Diego, California, p. 701 (1997).

7. M. POIER et al., Proc. 17th IEEE/NPSS Symp. Fusion Engineering, San Diego, California, p. 357 (1997).

8. O. NEUBAUER and S. NOWAK, Fusion Eng. Des., 58-59, 53 (2001).

9. M. POIER et al., Proc. 17th IEEE/NPSS Symp. Fusion Engineering, San Diego, California, p. 357 (1997).

10. B. GIESEN et al., Fusion Eng. Des., 69, 743 (2003).

11. W. ENGELHARDT and W. FENEBERG, J. Nucl. Mater., 76-77, 518 (1978). 
12. W. FENEBERG and G. H. WOLF, Nucl. Fusion, 21, 669 (1981).

13. T. E. EVANS, J. S. DEGRASSIE, and H. R. GARNER, J. Nucl. Mater., 162-164, 636 (1989).

14. S. C. McCOOL et al., Nucl. Fusion, 30, 167 (1990).

15. S. C. McCOOL et al., Nucl. Fusion, 29, 547 (1989).

16. A. GROSMAN, T. E. EVANS, and PH. GHENDRIH, Plasma Phys. Control. Fusion, 32, 1011 (1990).

17. C. DE MICHELIS, A. GROSMAN, and X. GARBET, Nucl. Fusion, 35, 1133 (1990).

18. M. KOBAYASHI et al., Nucl. Fusion, 40, 181 (2000).

19. M. KOBAYASHI, H. KOJIMA, K. ZHAI, and S. TAKAMURA, Phys. Plasmas, 7, 3288 (2000).

20. S. TAKAMURA, H. YAMADA, and T. OKUDA, Nucl. Fusion, 28, 183 (1988).

21. S. TAKAMURA, Y. KIKUCHI, Y. UESUGI, and M. KOBAYASHI, Nucl. Fusion, 43, 393 (2003).

22. M. S. FOSTER, S. C. McCOOL, and A. J. WOOTON, Nucl. Fusion, 35, 329 (1995).

23. K. H. FINKEN, Trans. Fusion Technol., 33, 291 (1998).

24. K. H. FINKEN, TH. EICH, and S. S. ABDULLAEV, Physicalia Mag., 20, 159 (1998).

25. K. H. FINKEN et al., Probl. At. Sci. Technol., 1(1), 2(2), 80 (1999).

26. K. H. FINKEN and TH. EICH, Contrib. Plasma Phys., 40, 57 (2000).

27. K. H. FINKEN et al., J. Plasma Fusion Res., 5, 77 (2002).

28. K. H. FINKEN, Trans. Fusion Sci. Technol., 41, 337 (2002).

29. M. JAKUBOWSKI, S. S. ABDULLAEV, K. H. FINKEN, and M. KOBAYASHI, Europhysics Conference Abstracts, 26B, 3.202 (2002).

30. M. W. JAKUBOWSKI et al., Probl. At. Sci. Technol., Series: Plasma Phys., 7, 42 (2002).

31. S. S. ABDULLAEV, K. H. FINKEN, and K. H. SPATSCHEK, Phys. Plasmas, 6, 153 (1999).

32. J. A. LICHTENBERG and M. A. LIEBERMANN, "Regular and Stochastic Motion," Applied Mathematical Sciences, p. 38, SpringerVerlag, New York (1983).

33. T. E. EVANS et al., J. Nucl. Mater., 145-147, 812 (1987).

34. A. GROSMAN et al., J. Nucl. Mater., 196-198, 59 (1992).

35. S. TAKAMURA et al., Phys. Fluids, 30, 144 (1987).

36. S. S. ABDULLAEV, K. H. FINKEN, A. KALECK, and K. H. SPATSCHEK, Phys. Plasmas, 5, 196 (1998).

37. S. S. ABDUllaEV, J. Phys. A, 32, 2745 (1999).

38. S. S. ABDUllaEV, J. Phys. A, 35, 2811 (2002).

39. K. H. FINKEN, S. S. ABDULLAEV, A. KALECK, and G. H. WOLF, Nucl. Fusion, 39, 637 (1999).

40. S. S. ABDUlLAEV, TH. EICH, and K. H. FINKEN, Phys. Plasmas, 8, 2739 (2001).

41. S. S. ABDULlAEV et al., Czech. J. Phys., 48, Suppl. S2, 319 (1998).

42. O. FISCHER and W. A. COOPER, Plasma Phys. Rep., 24, 727 (1998).

43. O. FISCHER, W. A. COOPER, and L. VILLARD, Nucl. Fusion, 40, 1453 (2000).
44. M. Z. TOKAR, Phys. Plasmas, 6, 2808 (1999).

45. A. KALECK, Contrib. Plasma Phys., 39, 367 (1999).

46. S. S. ABDULLAEV and K. H. SPATSCHEK, Phys. Rev. E, 60, R6287 (1999).

47. S. S. ABDULLAEV et al., Nucl. Fusion, 43, 299 (2003).

48. S. S. ABDULLAEV and K. H. FINKEN, Phys. Plasmas, 9, 4193 (2002).

49. PH. GHENDRIH, A. GROSMAN, and H. CAPES, Plasma Phys. Control. Fusion, 38, 1653 (1996).

50. K. H. FINKEN, TH. EICH, and A. KALECK, Nucl. Fusion, 38, 515 (1998).

51. TH. EICH, D. REISER, and K. H. FINKEN, Nucl. Fusion, 40, 1757 (2000).

52. K. H. FINKEN et al., J. Nucl. Mater., 266-269, 495 (1999).

53. TH. EICH, D. REISER, and K. H. FINKEN, J. Nucl. Mater., 290-293, 849 (2001).

54. A. M. RUNOV et al., Phys. Plasmas, 8, 916 (2001).

55. M. KOBAYASHI, TH. EICH, S. S. ABDULLAEV, and K. H FINKEN, IEEE Trans. Plasma Sci., 30, 66 (2002).

56. M. KOBAYASHI et al., Contrib. Plasma Phys., 42, 163 (2002).

57. K. H. FINKEN, Nucl. Fusion, 39, 707 (1999).

58. K. H. FINKEN et al., Czech. J. Phys., 49, Suppl. S3, 93 (1999). 59. K. H. FINKEN et al., Nucl. Fusion, 41, 503 (2001).

60. T. H. JENSEN, Fusion Eng. Des., 37, 437 (1997).

61. I. M. PANKRATOV, A. YA. OMELCHENKO, and V. V. OLSHANSKY, Probl. At. Sci. Technol., Series: Plasma Phys., 8, 5 (2002).

62. Y. KIKUCHI, Y. UESUGI, S. TAKAMURA, and A. G. ELFIMOV, Europhysics Conference Abstracts, 27A, P2.119 (2003).

63. I. M. PANKRATOV, A. YA. OMELCHENKO, and V. V. OLSHANSKY, Europhysics Conference Abstracts, 27A, P2.137 (2003).

64. D. W. FAULCONER and R. KOCH, Fusion Eng. Des., 37, 399 (1997).

65. K. H. FINKEN et al., Europhysics Conference Abstracts, 27A, O4.4Apd (2003).

66. M. KOBAYASHI et al., Europhysics Conference Abstracts, 27A, P3.168 (2003).

67. K. H. FINKEN et al., Plasma Phys. Control. Fusion (submitted for publication).

68. G. FREDERICI et al., Nucl. Fusion, 42, 1967 (2001).

69. K. H. FINKEN et al., J. Nucl. Mater., 290-293, 1064 (2001).

70. K. H. FINKEN, A. KRAEMER-FLECKEN, M. LEHNEN, and A. SAVTCHKOV, J. Nucl. Mater., 313-316, 1247 (2003).

71. S. J. FIELDING et al., Europhysics Conference Abstracts, 25A, P5.014 (2001).

72. A. GROSMAN et al., J. Nucl. Mater., 313-316, 1314 (2003).

73. T. SHOJI et al., Europhysics Conference Abstracts, 14B, 1452 (1990).

74. T. E. EVANS et al., Phys. Rev. Lett., 92235003 (2004).

75. T. E. EVANS et al., J. Nucl. Mater. (to be published).

76. S. S. ABDULLAEV and K. H. FINKEN, Nucl. Fusion, 38, 531 (1998). 